\title{
Utilización de coronas de zirconia como alternativa en la restauración de la caries en la temprana edad A propósito de dos casos clínicos
}

\author{
Dr. Walter Aníbal López Álvarez ${ }^{1}$ \\ Dr. Andrey Javier Türk Juárez ${ }^{2}$
}

\section{Resumen}

La caries dental es el resultado de un desbalance de múltiples factores, "Caries de la temprana edad» es el nombre que se le ha otorgado recientemente a las caries presentadas en piezas dentales en niños, principalmente se han observado en dientes anteriores primarios. Anteriormente se le conocía como "Caries de biberón», pero según se ha observado a lo largo del tiempo esta no es la única causa de su aparición, pues se ha notado que la leche materna a libre demanda y la utilización de chupetes son factores asociados a esta enfermedad (1, pp. 313-316).

El microorganismo asociado a la caries dental es el Streptococomutans, este coroniza la cavidad dental de los niños en cualquier momento de la vida, por lo cual se recomienda tomar medidas preventivas para mejorar los niveles de este microorganismo, estas pueden ser: mejorar la higiene oral, la dieta del niño (reduciendo alimentos y bebidas azucaradas). De igual manera es importante que el odontólogo mantenga un contacto con el pediatra, ya que este juega un papel importante, para proveer información sobre la salud oral del paciente.

Palabras clave: caries de la temprana edad, caries del biberón, Streptococomutans, higiene oral, pediatra.

\section{Abstract}

The dental decay has been known for decades as a multifactorial infectious disease. "Early childhood caries disease" is the term used recently that describes decay that affects primary dentitions in young kids. The first teeth to be affected are the upper primary anterior

\footnotetext{
${ }^{1}$ Dentista pediátrico, University of Pittsburgh (Clínica Dental Waly, S. A.)

2 Doctor asociado (estudiante de máster en Odontopediatría Integral y Ortopedia Dentofacial, Universidad Internacional de Catalunya (UIC)
} 
teeth which was first described as the "baby bottle syndrome". However, it seems that the bottle and the breastfeeding as well as the use of pacifiers are not the only cause associated with the disease. (1, pp. 313-316). The most common microorganism associated with the dental caries is the Streptococomutans. Usually it causes a cavitation on any surface of teeth during any moment of life. It is been recommended for years to implement different preventive methods to avoid higher levels of this microorganism, some of them includes improvement of oral hygiene, diet (less sugar). It is also advised and recommended that the pediatric dentist have a constant and open communication with the pediatrician because he's the one that has been in contact with the patients since their birth. They are the ones that can provide important information to the parents.

Keywords: Early childhood caries disease, baby bottle syndrome, Streptococomutans, oral hygiene, pediatrician. 


\section{Introducción}

La caries temprana es una enfermedad que se ve reflejada en los niños. La Revista Pediátrica de Atención Primaria, 1999, menciona que entre el $5 \%$ y el $8 \%$ de la población infantil se ve afectada, pero hay un incremento en aquellos pacientes pertenecientes a clase social baja o de etnias de menor población. Con frecuencia los padres no saben de la importancia de la dentición temporal y de su repercusión en el correcto desarrollo de la dentición permanente (5, pp. 1897-1904).

A partir de diferentes experiencias de atención al paciente se ha procurado restaurar estéticamente las piezas temporales afectadas por las caries, lo que ha sido una preocupación por décadas. Afortunadamente el advenimiento de la utilización de zirconia en coronas pediátricas, representa una excelente alternativa para restaurar y retomar la función y la estética anterior (3, pp. 431-439).

Las caries de la temprana edad se caracterizan por la afección de los dientes superiores anteriores y los molares inferiores predominantemente, aunque en casos severos pueden afectarse el resto de las piezas temporales (4, pp. 1897-1904).

Las caries de la temprana edad han mostrado dos factores clave, el primero de ellos es la secuencia de erupción de las piezas dentales, principalmente afecta a los dientes anterosuperiores; y el segundo se le atribuye a la ubicación de la lengua, durante la alimentación, ya que es esta la protectora de los dientes inferiores de los alimentos cariogénicos, por esta razón estos dientes no se encuentran afectados frecuentemente. (5, pp 32-44).

\section{Coronas de zirconia como} alternativa de tratamiento

El zirconio es un material que se utiliza en odontología desde hace veinte a veinticinco años aproximadamente, y en los últimos diez ha supuesto una auténtica revolución, gracias a sus inmejorables propiedades estéticas y funcionales.

El zirconio es el material idóneo para realizar coronas dentales de alta calidad estética. Además, es un material totalmente biocompatible con el organismo, ya que al no llevar metal (níquel, cromo o cobalto), evitamos el riesgo de alergias en pacientes predispuestos.

Gracias a su alta transparencia y color blanco conseguimos una corona dental con un tono natural que imita nuestros dientes, por lo que no se nota diferencia alguna. Siempre se mantendrá la homogeneidad del color del diente elegido.

La corona de zirconia se integra perfectamente con la encía, creciendo el tejido hasta el diente, igual que en una pieza natural, otro beneficio es su baja adherencia de placa dental, al ser comparada con las coronas de metal.

La conductividad térmica que tiene la corona de zirconia da la posibilidad de poder consumir alimentos fríos o calientes sin tener sensaciones desagradables por las temperaturas.
Ventajas de las coronas de zirconia en los niños (6)

- Estética para competir con coronas fabricadas en laboratorios personalizados.

- Durabilidad demostrada en odontología para adultos durante más de diez años.

- Buena alternativa para pacientes sensibles al níquel.

- Protección completa de la pieza dental.

- Tiempo mínimo de silla.

- Reducción dental similar a coronas de acero (se necesitará una reducción más circunferencial).

Desventajas de las coronas de zirconia en niños (6)

- El diente debe estar preparado para ajustarse a la corona.

- La saliva y la hemorragia deben ser controladas.

- Curva de aprendizaje.

\section{Materiales y métodos}

Los casos clínicos corresponden a un paciente de cuatro años de edad y uno de cinco, con caries de la temprana edad, con una evolución de dos y tres años respectivamente, en los cuatro incisivos anteriores. Las restauraciones finales fueron con coronas de zirconia. 
$\frac{\pi}{c} \overbrace{0}^{0}$ Descripción de la técnica

1. Con una fresa de carburo o diamante cónico grueso recortar 1.5-2 $\mathrm{mm}$ del borde incisal y romper los contactos interproximales

Figura 1

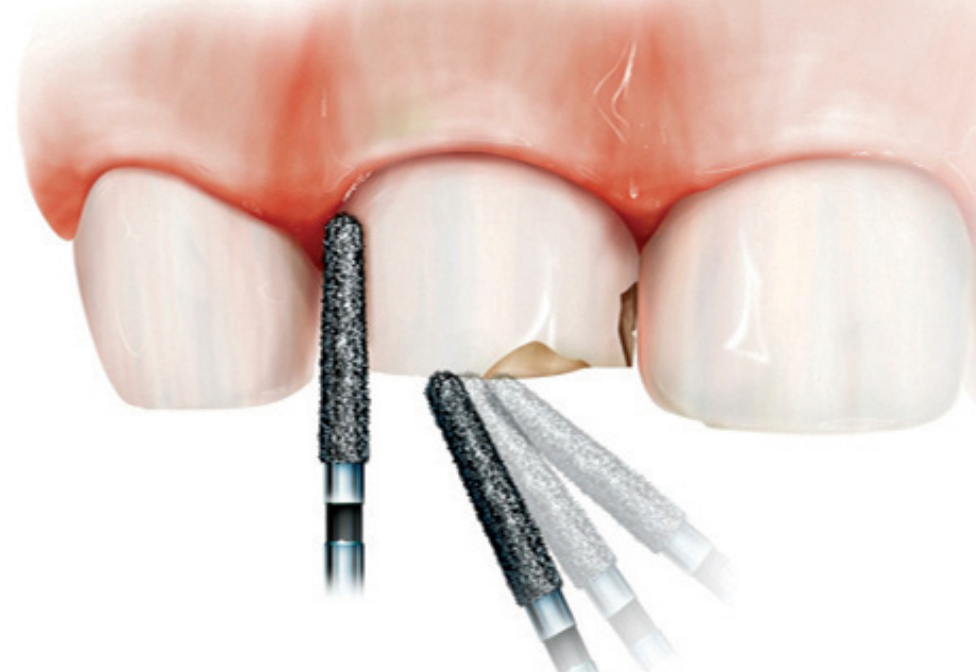

Fuente: con autorización de NuSmile, Ltd. Copyright, 2018.

2. Cuidadosamente prepare el diente supra gingivalmente para evitar la laceración del tejido

Figura 2

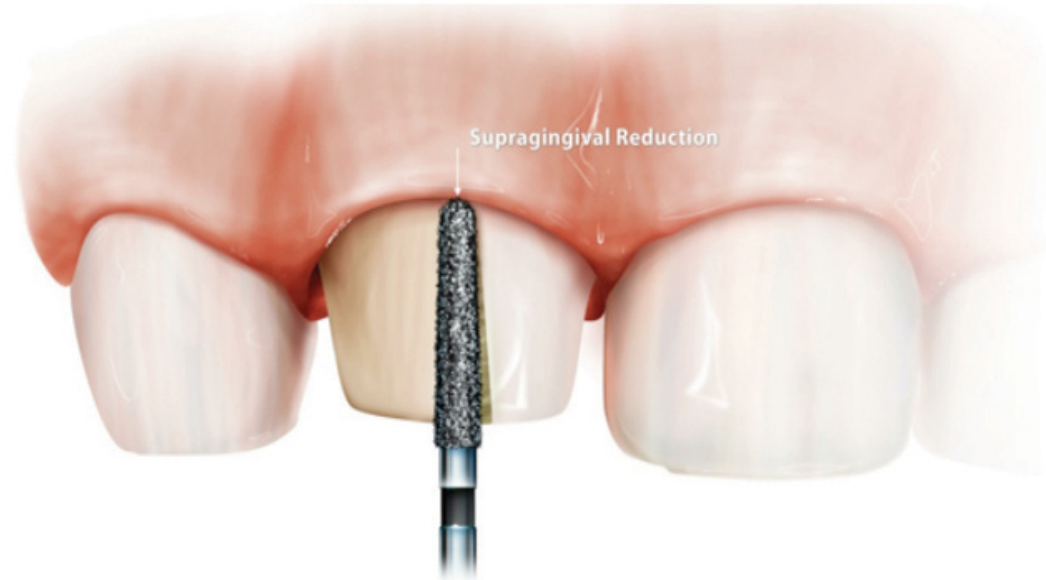

Fuente: con autorización de NuSmile, Ltd. Copyright, 2018. 
3. Use un diamante cónico fino para crear un margen de borde de pluma de 1 a $2 \mathrm{~mm}$ de forma subgingival

Figura 3

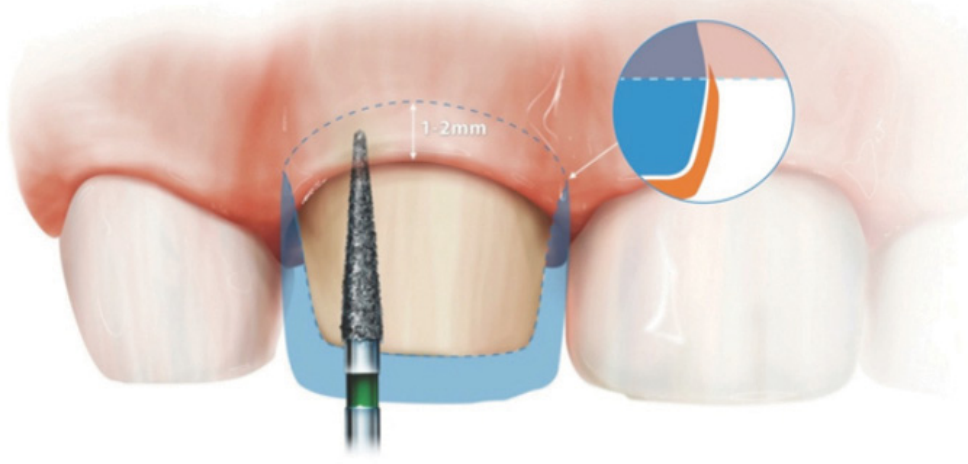

Fuente: con autorización de NuSmile, Ltd. Copyright, 2018.

4. Dejar los ángulos redondeados, hacer una reducción total en el diente de aproximadamente $20-30 \%$

Figura 4

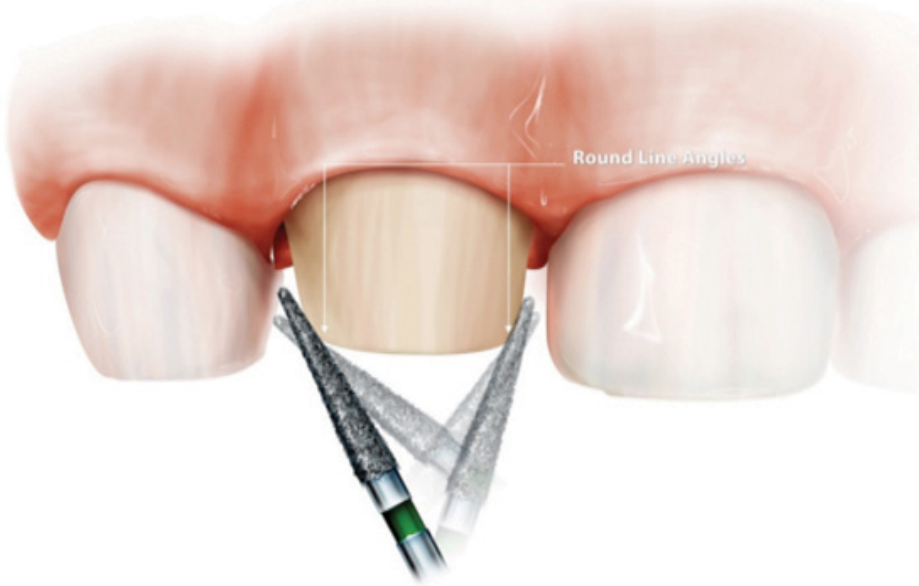

Fuente: con autorización de NuSmile, Ltd. Copyright, 2018.

5. Ajuste de prueba con corona Try-In rosado

Figura 5

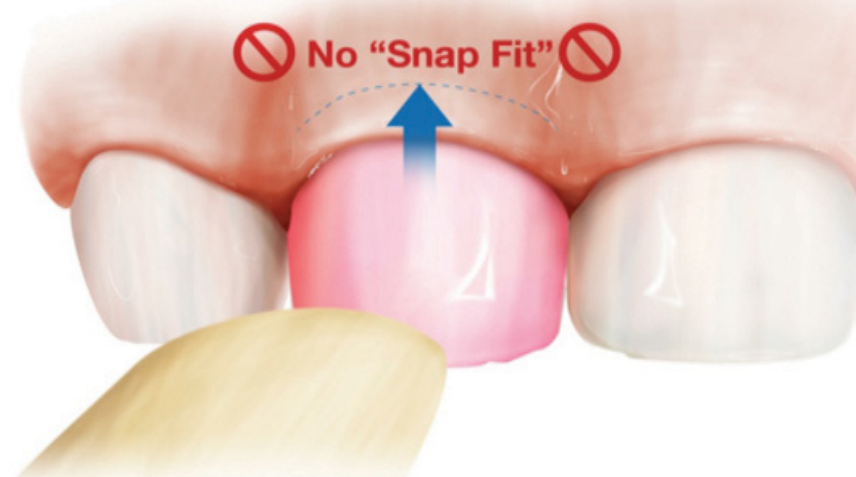

Fuente: con autorización de NuSmile, Ltd. Copyright, 2018. 
6. Verificar el ajuste y la alineación

Figura 6
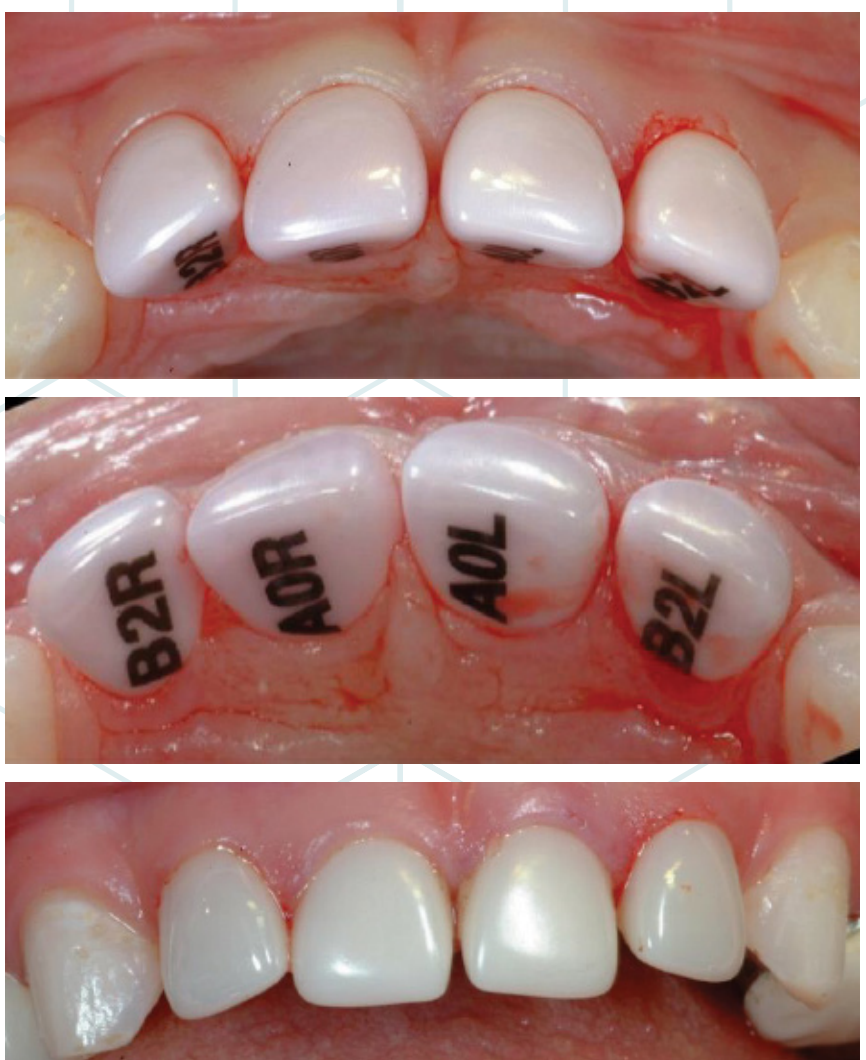

Fuente: con autorización de NuSmile, Ltd. Copyright, 2018.

7. Cementar la corona con BioCem, Ceramir, Cemento de resina, o RMGI

Figura 7

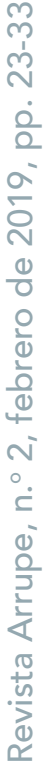

Fuente: con autorización de NuSmile, Ltd. Copyright, 2018. 


\section{Figura 8}

Fuente: con autorización de NuSmile, Ltd. Copyright, 2018.

9. Fotocurar cuando corresponda y comenzar la limpieza final Figura 9

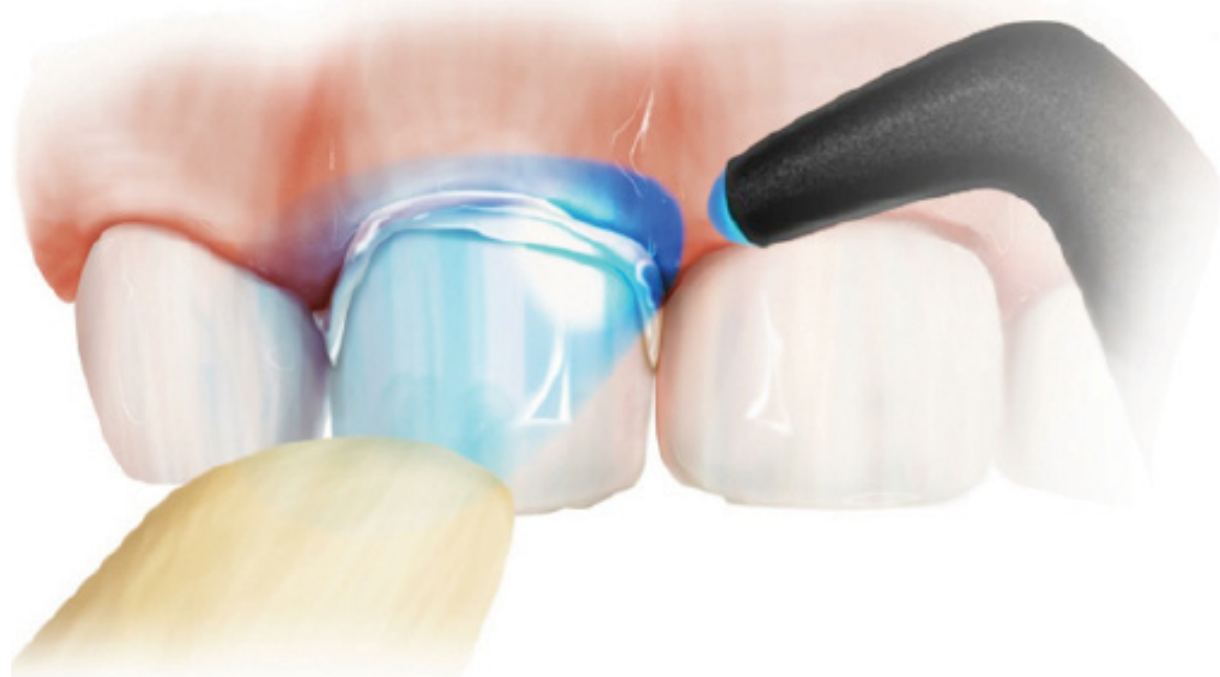

Fuente: con autorización de NuSmile, Ltd. Copyright, 2018. 
Figura 10

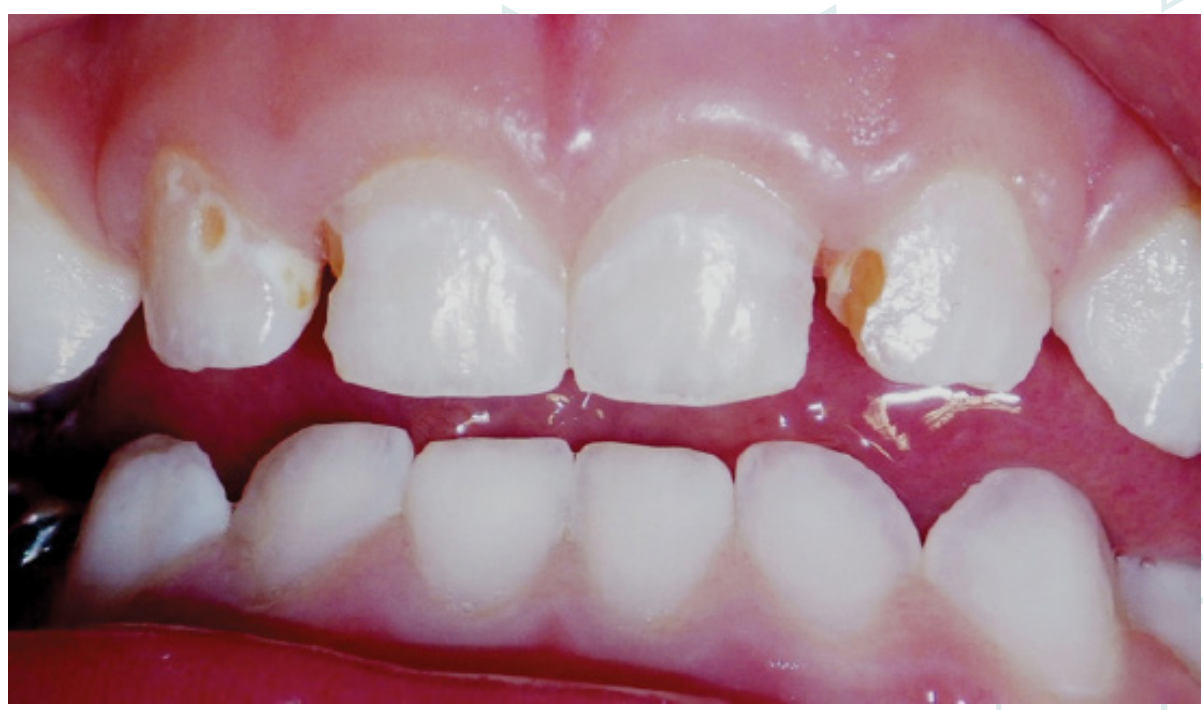

Fuente: con autorización de NuSmile, Ltd. Copyright, 2018.

Preparaciones dentales

Figura 11

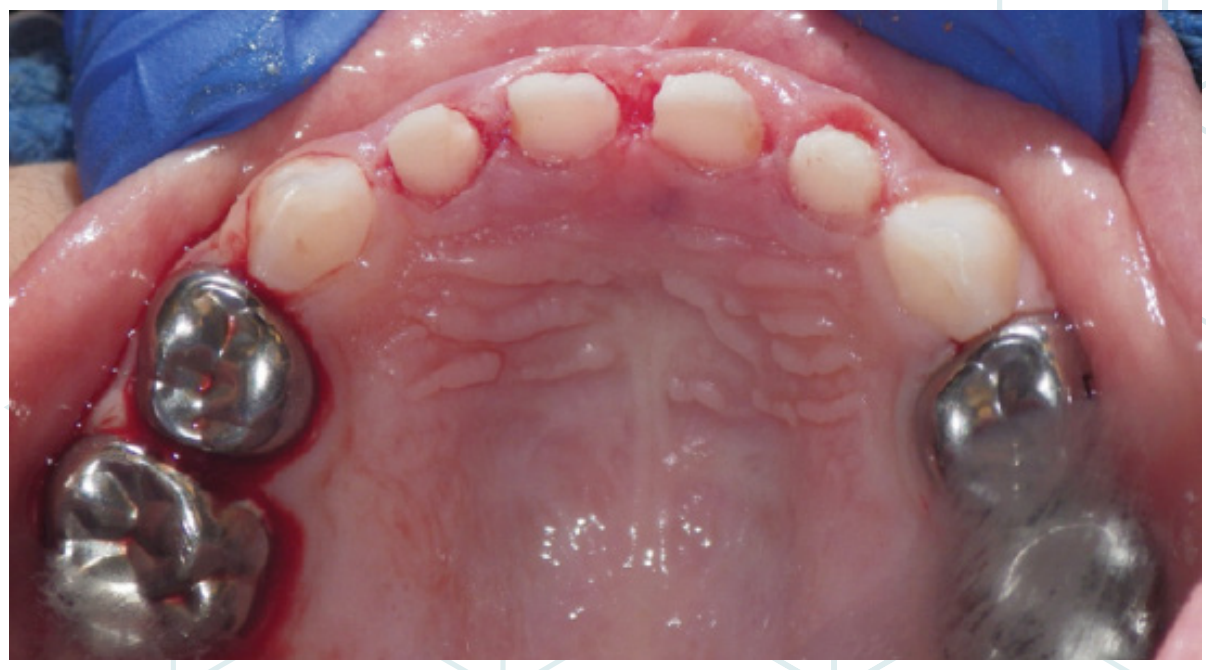

Fuente: con autorización de NuSmile, Ltd. Copyright, 2018. 
Figura 12

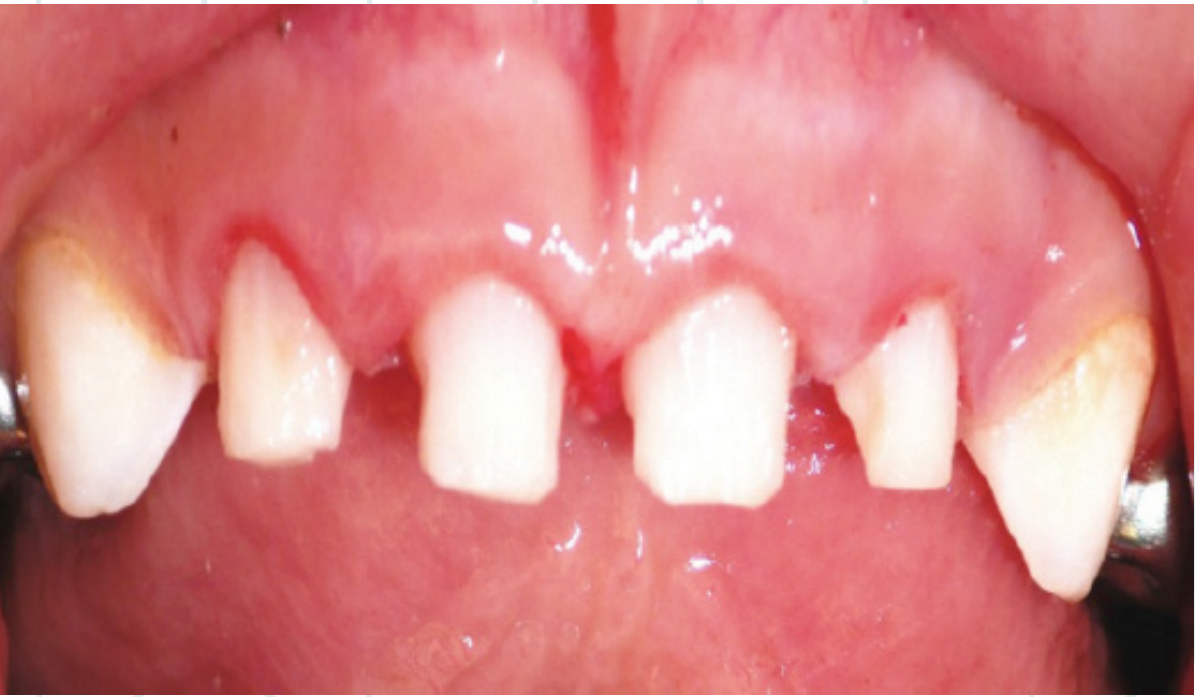

Fuente: con autorización de NuSmile, Ltd. Copyright, 2018.

\section{Postoperatorio una semana}

Figura 13

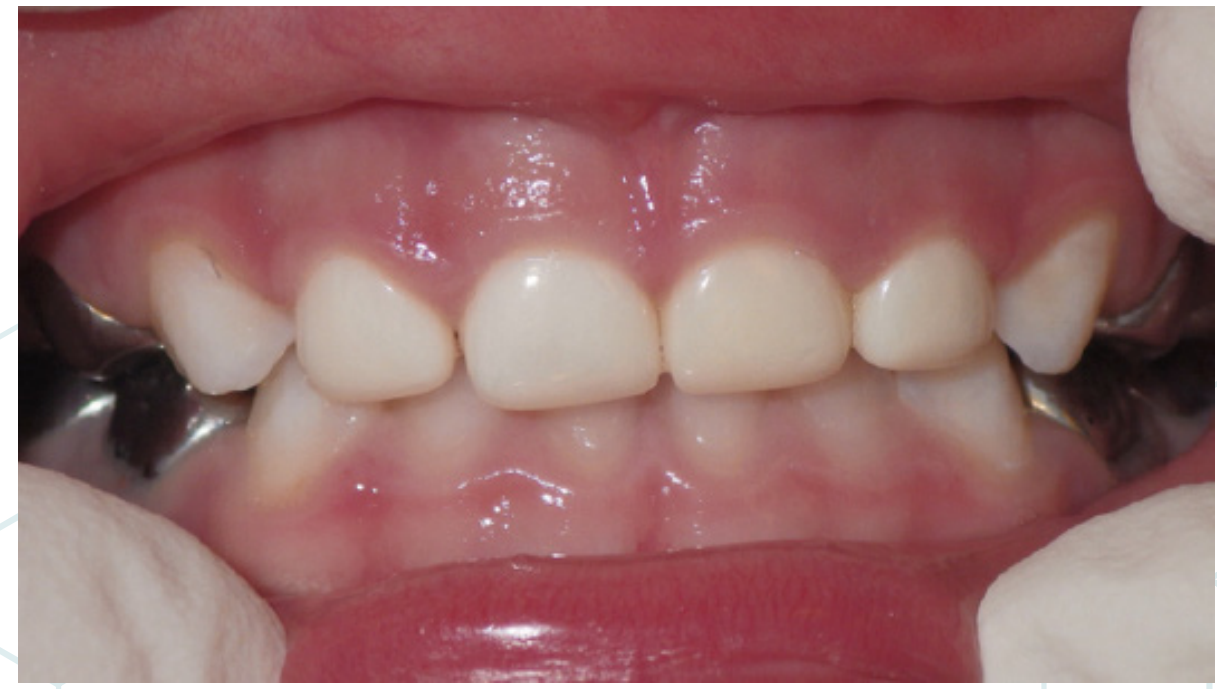

Fuente: con autorización de NuSmile, Ltd. Copyright, 2018. 


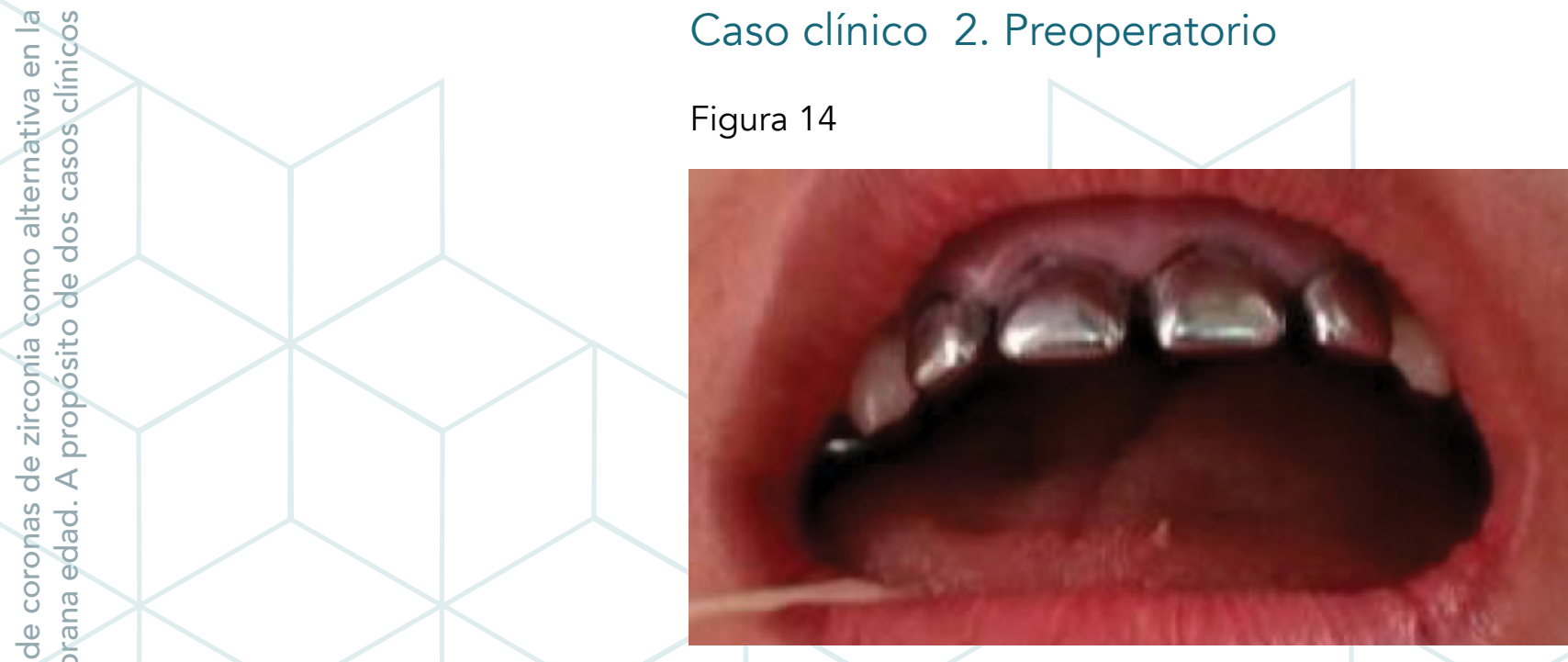

Fuente: Clínica Dental Privada Waly, S. A., 2018.

Preparaciones dentales

Figura 15

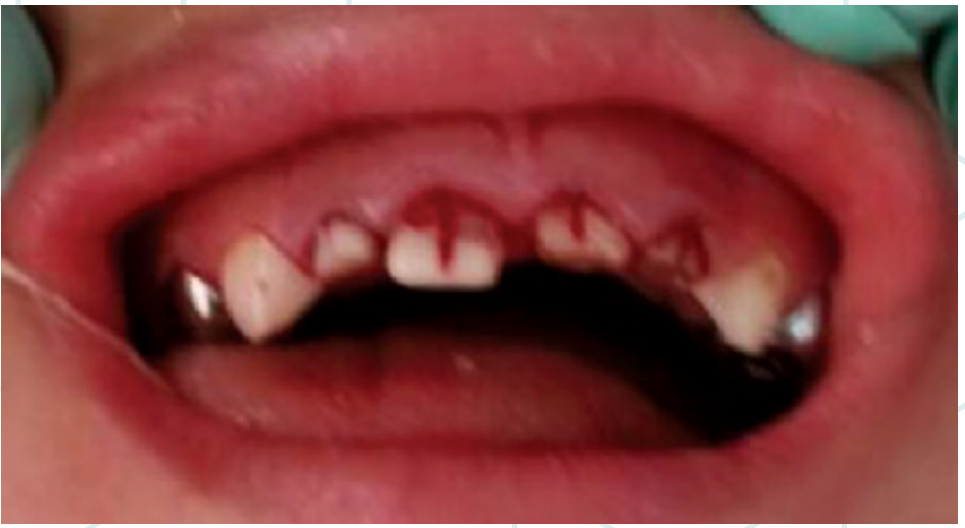

Fuente: Clínica Dental Privada Waly, S. A., 2018.

\section{Cementado}

\section{Figura 16}

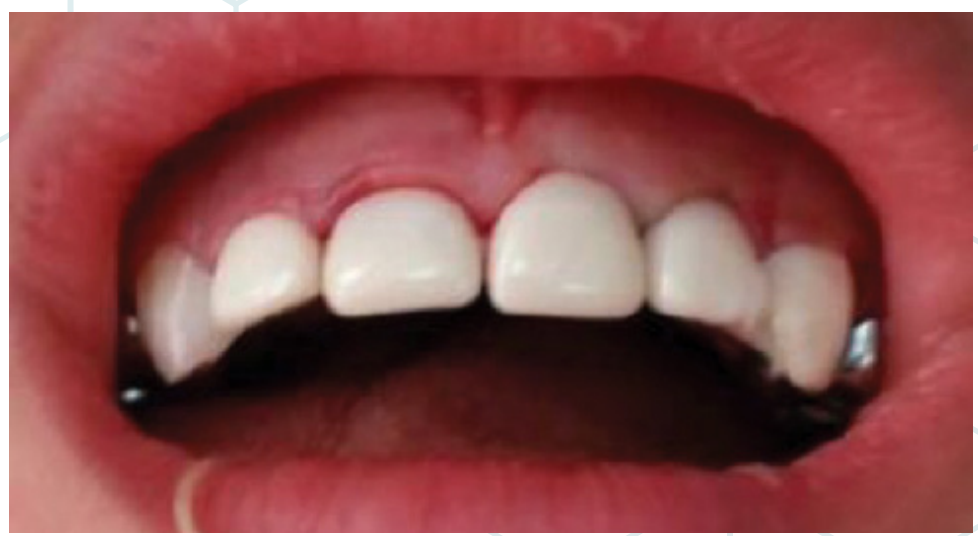


Después de cementadas las coronas de zirconia se pudo observar una rápida recuperación del tejido gingival con poca inflamación. Luego de una semana, durante la evaluación, se observó que el tejido gingival se adaptó perfectamente. Se observó una recuperación más rápida en las coronas de zirconia que las que se tiene con las coronas de acero convencionales.

\section{Conclusión}

El tejido gingival tuvo un resultado de adaptación de primer nivel, pues se observó que la recuperación del paciente es más efectiva implementando las coronas de zirconia, en comparación con las coronas convencionales de acero. Las coronas de zirconia son el tratamiento a seleccionar cuando los objetivos son más inclinados hacia la estética en los dientes anteriores.

\section{Referencias}

1. Ayhan H. Influencing factors of nursing caries. J. Clin Pediatr Dent 1996; 20(4): 313-316.

2. American Academy of Pediatric Dentistry. Policy on early childhood caries (ECC): Classifications, consequences, and preventive strategies. Pediatr Dent 2007/2008; 29 (suppl): 39-41.

3. Filstrup SL, Briskie D, Fonseca M. Early childhood caries and quality of life: Child and parent perspectives. Pediatr Dent. 2003; 25(5): 431-439.

4. Planells del Pozo P. Prevención en odontopediatría. En: Tratado de Odontología. Madrid: Smithkline Beecham, 1998; 1897-1904.

5. Reisine S, Douglass JM. Psychosocial and behavioral issues in early childhood caries. Community Dentistry \& Oral Epidemiolgy 1998; 26(1): 32-44.

6. Casamassimo P, Hughes C, Stamper J, Waggoner W, NuSmile Corporation, 2012. [internet ]. Houston TX. Recuperado a partir de: https://www.nusmile.com 